\title{
A Hybrid CNN- GLCM Classifier for Detection and Grade Classification of Brain Tumor
}

\author{
Akila gurunathan ( $\nabla$ akilapg123@gmail.com ) \\ PSNA CET: PSNA College of Engineering and Technology https://orcid.org/0000-0002-2211-2009

\section{Batri Krishnan} \\ PSNA College of Engineering and Technology
}

\section{Research Article}

Keywords: Convolutional neural network (CNN), classification, segmentation, meningioma

Posted Date: June 7th, 2021

DOI: https://doi.org/10.21203/rs.3.rs-531022/v1

License: (9) This work is licensed under a Creative Commons Attribution 4.0 International License. Read Full License

Version of Record: A version of this preprint was published at Brain Imaging and Behavior on January 19th, 2022. See the published version at https://doi.org/10.1007/s11682-021-00598-2. 


\section{A hybrid CNN- GLCM classifier for detection and grade classification of brain tumor}

$1^{*}$ Akila Gurunathan and ${ }^{2}$ Batri Krishnan

${ }^{1}$ Assistant Professor, Department of Electronics and Communication Engineering, PSNA College of Engineering and Technology, Dindigul , Tamilnadu , India. E-mail: akilapg@gmail.com

${ }^{2}$ Professor, Department of Electronics and Communication Engineering, PSNA College of Engineering and Technology, Dindigul , Tamilnadu, India. E-mail: Krishnan.batri@gmail.com

*Corresponding author

A R T I C L E I N F O

\section{Keywords:}

Convolutional neural network $(\mathrm{CNN})$, classification, segmentation, meningioma.
A B S T R A C T

Early identification and diagnosis of brain tumor using a supervised approach plays an essential role in the field of medicine. In this paper, an automated computer-aided method using deep learning architecture named CNN Deep net is proposed for the detection, classification and diagnosis of meningioma brain tumor. This proposed method includes preprocessing, classification, and segmentation of the primary occurring brain tumor in adults. The proposed CNN Deep Net architecture extracts the features internally from enhanced image and classifies them into normal and abnormal tumor images. The segmentation of tumor region is performed by the global thresholding with area morphological function method. This proposed method of fully automated classification and segmentation of brain tumor preserves the spatial invariance and inheritance. Furthermore, based on its feature attributes the proposed CNN Deep net classifier, classifies the detected tumor image belongs either to (low grade)benign or (high grade)malignant. This proposed CNN Deep net classification methodology approach with grading system is evaluated both quantitatively and qualitatively. The quantitative measures such as sensitivity, specificity, accuracy, Dice similarity coefficient, precision, F-score of the proposed classification and segmentation methodology states a better segmentation accuracy and classification rate of $99.4 \%$ and $99.5 \%$ with respect to ground truth images. 


\section{Introduction}

A tumor is a volume of irregular and abnormal cells affecting the function of nearby healthy cells in a human body. Meningioma is the most commonly occurring tumor in adults with high risk factors. Meningiomas are seen in the dura mater, which are the outer tissues of the brain and called meninges. They are mostly extra-axial neoplasms, and only a few are intracranial tumors. The general classification of tumor is Benign or noncancerous tumor and Malignant or cancerous tumor. Benign does not spread to other parts of the body and does not generate new tumor. Malignant or cancerous tumor appears in great mass and pulls out healthy cells by taking nourishment from body tissues. Malignant tumors are fast in their growth, and their rate of reoccurrence is high even after the surgery. These tumors spread to other parts of the body through the lymphatic blood vessels. It is observed that 70 percent of the tumors are benign and are categorized as Grade I. The Grade I benign tumor has less amount of cancerous cell multiplication rate which is specified as mitotic rate(HPF), and is always less than 4 mitoses per HPF. The Grade I have nine subtypes. A typical Meningioma -Grade II occupies 22-26 percent of the occurrence in the total tumor occurrence percentage rate. They have a tumor cell growth rate of 15 mitoses per HPF. Anaplastic -Grade III is dangerous, and it occurs in fewer numbers. But the mitotic rate of Grade III is higher than 18 and patients are at a high risk of death. In general, the diagnosis of brain tumor begins with magnetic resonance imaging (MRI) as it provides detailed information on both hard and soft tissues with fat and fluid substances of the brain through electromagnetic fields when compared to other imaging modalities like CT, $\mathrm{X}$ ray, PET etc. A specific pattern or a regular range of radiation intensity is obtained when human brain is exposed to radiation by different modalities. The NCIS and WHO has reported that every year around 13,000 people are affected by the tumor. Every year the death rate is progressively increasing because of the late diagnosis and semi-automated grading system. The detection and classification of tumors by manual method is the greatest challenge which pathologist's face. According to the reports published in Cancer.Net and WHO 2019, automated classifier architecture for the detection and classification of tumor is an emerging research area in the field of medicine. This provokes number of researchers to develop a cost effective as well as more precise automated algorithm for detection, classification and diagnosis of tumor.

After the emergence of machine learning techniques in medical imaging analysis the detection and classification approaches has become easier and effective in terms of accuracy. An image analysis scheme of CNN Deep Net is proposed for the detection and classification of meningioma tumors with high accuracy and classification rate. This article uses the standard medical image processing sub modules such as preprocessing, feature extraction, classification and segmentation for detection and localization of tumor regions in brain MR images.In this regard ,the article is organized as follows: In section 2 ,various existing brain tumor classification and segmentation approaches using deep learning ,machine learning are discussed. In section 3, novel brain tumor detection, classification and segmentation methodology is proposed and section 4 discusses the results of the proposed tumor method. Finally, section 5 concludes the work with future scope. 


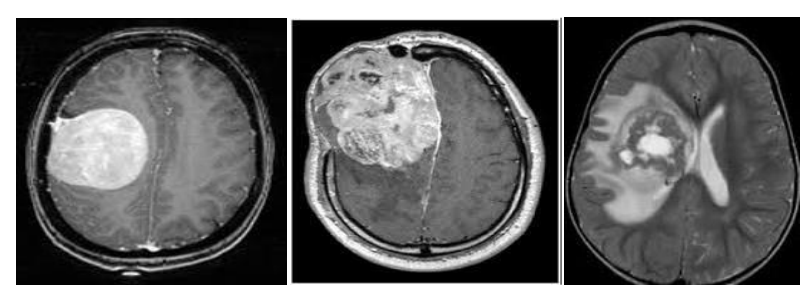

Fig. 1. a) Brain Tumor MR image b) benign c) malignant meningioma

\section{Literature Survey}

Sheela et al. (2020) used fuzzy C means algorithm in detecting the tumor affected regions on the detection and morphological segmentation of tumor regions are used in brain MRI images for segmentation purpose. Sun et al (2019) presents a 3D CNN architectures and deep learning based framework for brain tumor segmentation. In addition to it, survival prediction using multimodal MRI scans is presented. Decision tree based classification with cross validation is performed and thus framework reduces model bias and extracted 4524 features. A random forest approach is used for prediction of survival and achieves $61 \%$ accuracy on short, mild and long survivals. Sajid et al proposes a deep learning models using long short term memory (LSTM) and convolutional neural networks (ConvNet) for accurate brain tumor delineation from benchmark medical images. A combined ensemble of ConvNet and LSTM is presented with multiple combinations of preprocessing and class weighting model. This model calculates Dice similarity score for a combination of ConvNet and LSTM ensemble model and achieves 82.29\%.Javaria Amin et al proposes a model to detect tumor at an early phase. In this method, Weiner filter is used for denoising with potential field clustering .Morphological segmentation is performed in FLAIR and T2 MRI images and features are extracted by fused LBP and GWT.The proposed model achieves 93\% precision and 96\% accuracy. Kai Roman Laukamp et al proposes multi parametric deep learning model for automatic detection and segmentation of meningioma. A voxel-wise classifications of four tumor classes defined in the BRATS benchmark is used in the model .The proposed model uses bias field correction ,registration and skull stripping as preprocessing techniques. Heba Mohsen al et al. (2017) proposes a network to identify affected tumor regions in brain using deep learning approaches. The method applies DWT for segmentation of detected image and uses PCA feature extraction method. This non-linear approach produced a significant simulation result of $96.7 \%$. Rajagopal (2019) proposes a ML approach to find the brain tumor regions by energy technique and segmented the affected regions. In addition, classification is also performed using random forest classification approach and achieves $98 \%$ accuracy. Abdelmajid Bousselham al et al (2019) uses reinforcement and enhancement methods to improve the performance of classification and segmentation of tumor region. The classification and segmentation were performed on the basis of the temperature mapping of tumor in MRI from its thermal information. Detection of tumor is done through canny edge detector.This approach of detection and segmentation on BRATS 2012 and 2013 are compared with chan vese based approach and shows significant results. Javaria Amin et al. (2019) proposed a statistical method for denoising and lesion enhancement using wiener filter with different wavelet bands. The Potential field clustering of tumor pixels with global thresholding is used for segmenting tumor regions where a local binary pattern and Gabor wavelet is used for feature extraction of brain tumor regions. Texture features helps to predict the tumor regions more 
accurately. This approach yields 92\% accuracy on BRATS 2013 and 2015 datasets. Amin Kabir Anaraki et al (2019) convolutional neural network with genetic algorithm is used for classification of glioma in MR images. In this method, networks with many layers and parameters are analyzed using bagging method. The accuracy rate of 94.2 $\%$ is achieved and reveals the effectiveness of CNN. Muhammad Sajjada et.al (2019) proposed a computer-aided diagnosis (CAD) system to assist radiologists in diagnosing tumor using CNN based on multi grade classifier. Segmentation of affected tumor region by deep learning technique is done and training of data is used for grade classification of tumors .A pre trained VGG $19 \mathrm{CNN}$ model is fine tuned for better classification. Pereira et al. (2016) uses popular DL technique called Convolutional neural network classifier for classifying the abnormal patterns in brain. This method produces high segmentation results of $90 \%$ with high resolution brain images. Khawaldeh, Saed et al. (2017) proposed a modified AlexNet for the detection and classifications of brain tumor images from non brain tumor images and obtain 91.6\% average classification accuracy. Hiba Mzoughi et al.(2020)proposes a deep multi -scale 3D Convolutional Neural Network (CNN) for MRI Gliomas Brain Tumor Classification. With validation dataset and data augmentation, the proposed approach achieves $96.49 \%$ accuracy.

\section{Contribution}

Machine learning approaches for the brain tumor classification requires large number of extracted features for efficient classification. Thus different ML approaches uses a separate feature extraction method and classifies the tumor from normal brain MRI image. These feature extraction and classification approaches are not fully automated. They require labeling of classes for classification. Moreover the conventional methods lack in achieving spatial inheritance and invariance. Hence a fully automated classification approach, CNN Deep net is proposed .This proposed architecture consists of five convolutional layers with ReLU activations for achieving nonlinearity and maxpooling layers for feature reduction along with three fully connected layer and a classification layer.The proposed work satisfies the requirement of large volume of dataset for deep learning classification methodology by using data augmentation. A combined fully automated feature extraction and classification is achieved through convolution and classification layers. The accuracy of the proposed CNN Deep net is improved by hyper tuning techniques such as normalization, regularization and dropout. Furthermore, a novel hybrid classification approach GLCM-CNN classifier is proposed to diagnosis the detected brain tumor either as low grade (benign) or high grade (malignant). Block diagram of the proposed CNN Deep net is shown in Fig.2. 


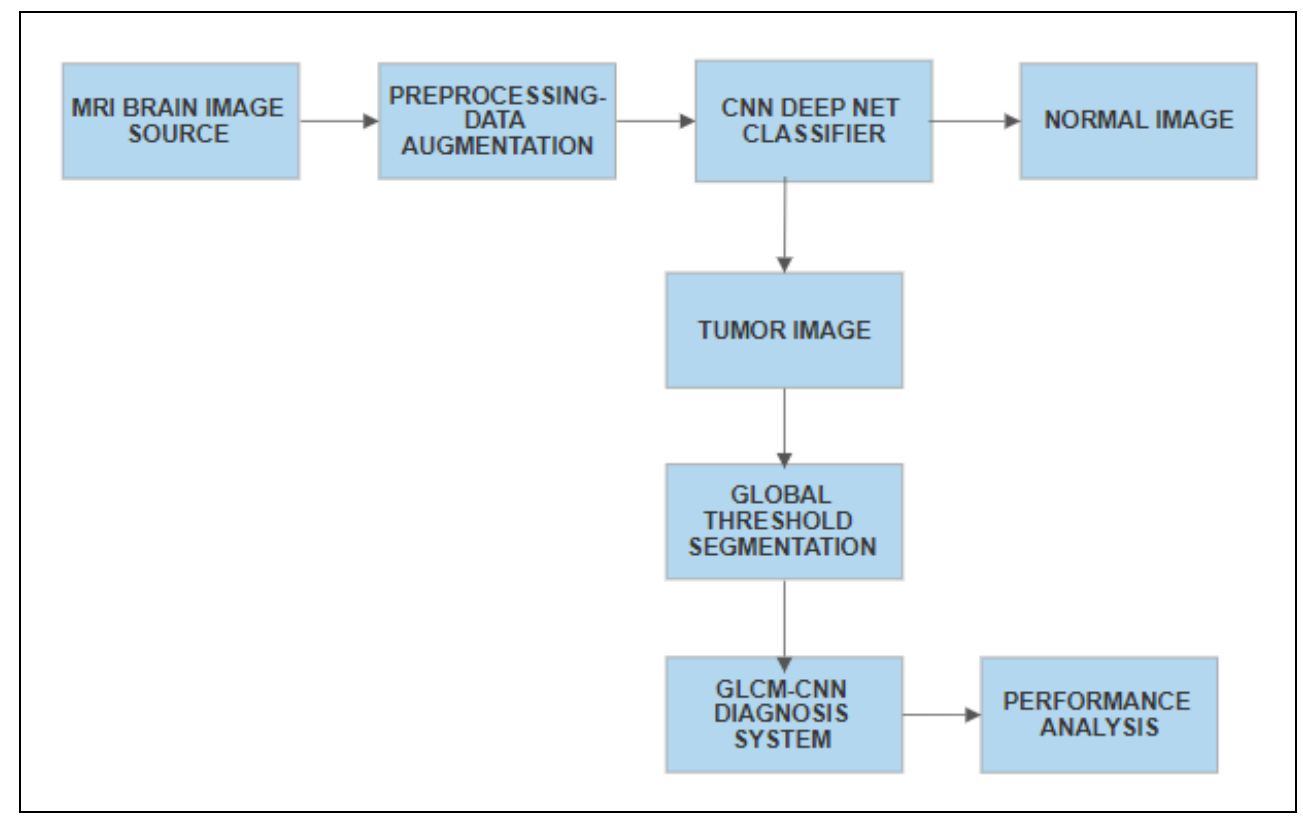

Fig. 2 Proposed brain tumor classification using CNN architecture

\section{Materials and Methods}

\subsection{Materials}

The performance of the proposed CNN Deep Net is observed on the MR brain images of open access BRATS datasets. This dataset includes ground truth images that are obtained from expert radiologist. This paper access 600 brain images from the dataset and it has both normal brain images (340 brain images) and abnormal brain images (260 brain images). This dataset is grouped into training and testing set. The training dataset contains 90 normal images and 75 abnormal images. The testing dataset contains 250 normal images and 185 abnormal MR images. The images used in the proposed work are T1 and T2 weighted sequence with contrast agent in axial position with 1000 slices per pixel resolution. As T1 weighted sequence shows better delineating anatomy and morphology, where T2 weighted images shows pathology to highlight the lesions well, in brain images.

\subsection{Methods}

The meningioma tumor prediction and classification in MR image is carried out using proposed CNN Deep net. All dataset images of 512*512 pixel resolution are resized into $256 * 256$ pixels and achieves same dimensionality with scaling range of [1 1.2] . This preprocessed brain MRI image is classified either into normal or abnormal using the proposed CNN Deep net classifier. The tumor regions are segmented using global thresholding approach in fusion with connected component method. A combined GLCM and CNN classifier is proposed in this paper for diagnosis of segmented regions.

\subsubsection{Preprocessing}

The preprocessing technique includes resizing of brain image sources to uniform dimensionality images with flipping up /down directions and rotating images at \pm angles and skewing etc through data augmenting. Thus data 
augmentation helps the proposed CNN Deep net architecture to attain a high accuracy and precision in classification. The data augmentation of the proposed CNN Deep net is listed in the Table 1. Fig.3 shows the augmented results of input brain MR images.

\begin{tabular}{|c|c|}
\hline Augmentation parameters & Value \\
\hline Rotation & 15 \\
\hline Width/height Shift & 0.05 \\
\hline Reflection & $1 / 0$ \\
\hline Horizontal /vertical flip & set \\
\hline Scale range & {$[11.2]$} \\
\hline Shear & 0.05 \\
\hline
\end{tabular}

Table 1: Data augmentation parameters of proposed CNN Deep Net architecture.

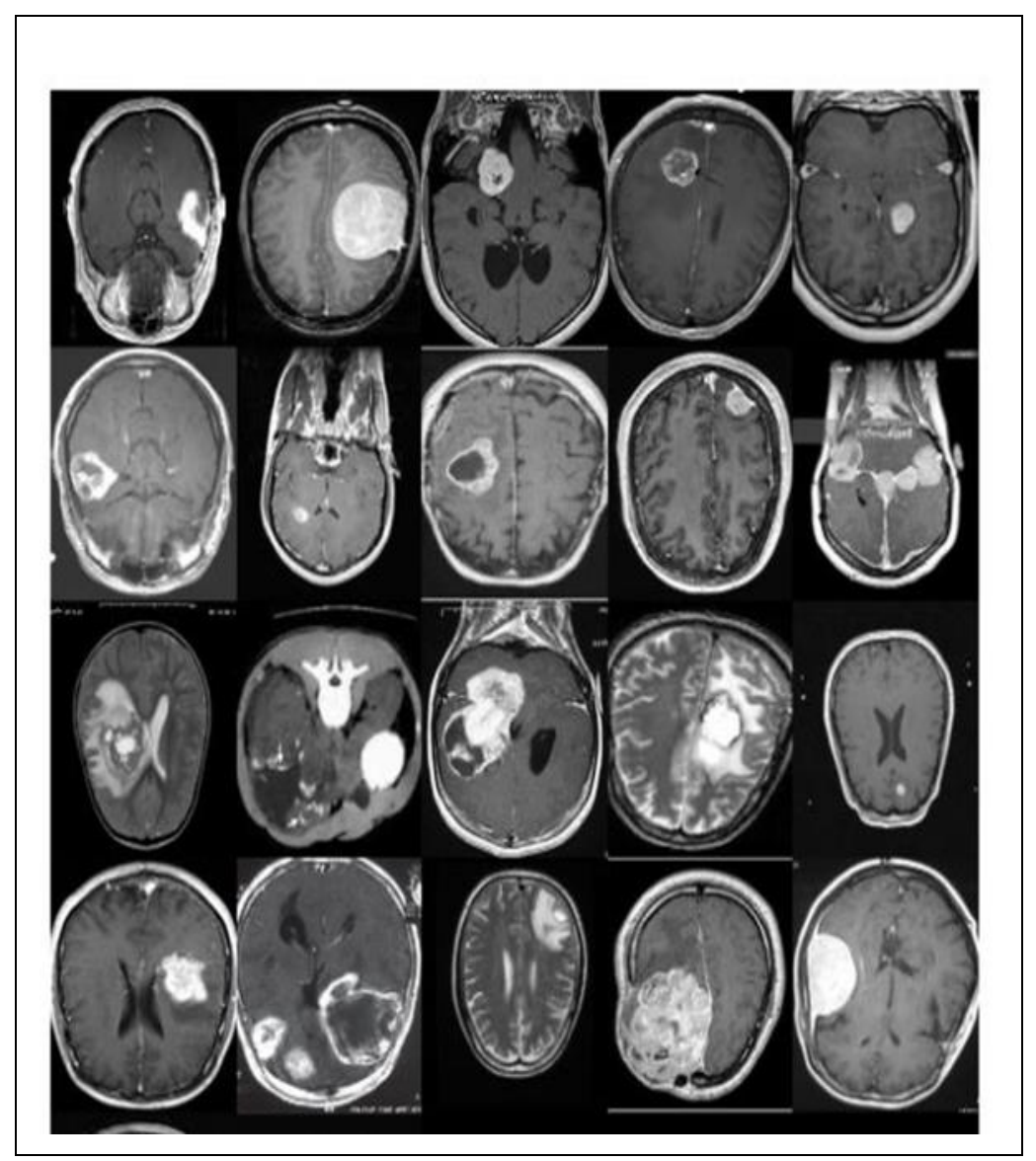

Fig3. Data Augmented outputs of brain MRI image

\subsubsection{Feature extraction and Classification}

On the basis of neural network's parameter sharing property, the parameters or features are reduced in number and hence network starts learning through indirect interactions in deep learning approach. An end to end learning technique is proposed with deep learning architecture where feature extraction and classification are combined 
together. Thus input images are learning automatically in deep learning architecture. This supervised deep learning representation uses both linear and non linear transformation with multiple layers in neural network to make prediction more accurate. A high speed performance analysis tool called convolutional neural network is used in the proposed methodology for the classification of 2D images. The structure of CNN is stated using convolution, max pooling, and fully connected layers. The entire internal architecture of CNN classifier is depicted in Fig. 4.

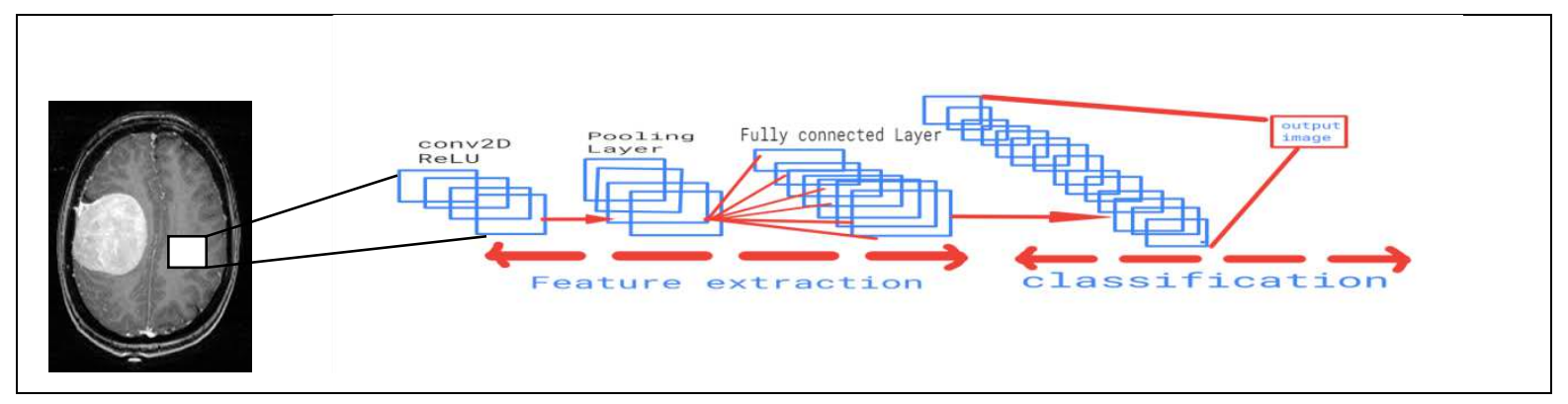

Fig. 4. Proposed CNN classifier architecture

\subsubsection{Convolutional Filters}

The convolutional layer focuses with both higher and lower level features namely edge detection, smoothening and sharpening etc. The convolution of an input image with a feature detector called kernel filter is carried out across the image through the sliding window method. As the depth of the filter is equal to the input image, the proposed method uses $3 \times 3$ kernel filter. An element wise multiplication is done and added up to give feature maps, which are the output of convolutional layers. The value of feature map is the value of matched input image and filter value .Hence the filter map is a 2D matrix. They are also known as activation maps or convolved features.

The convolution function is depicted in the following equation.

$$
\begin{gathered}
\mathrm{X}=\mathrm{u} * \mathrm{~V} \\
I * J(t)=\int I(\tau) J(\tau-1) d t
\end{gathered}
$$

The symbol * indicates convolution process and the equation represents percentage of filter $(\mathrm{J})$ area overlapping with input image (I) at a time $\tau$.

Applying the tighter bounds to the integral the equation can be rewritten as

$$
I * J(t)=\int_{0}^{t} I(\tau) J(\tau-1) d t
$$

The equation corresponds to single entry in $1 \mathrm{D}$ to compute complete convolution tensors . The multidimensional kernel tensors are required and it is given by,

$$
(I * J)(x, y)=\int_{0}^{x} \int_{0}^{y} I(x-i, y-j) H(i, j) d i . d j .
$$

The convolution slides the kernel over the input image .It is mathematically given by the above equation and the output of equation will be scalar value. This process is repeated for every xy pixels and results are stored in a convolutional matrix called feature maps. The main purpose of this convolutional layer is to reduce the size of input image for the upcoming fast process. The performance of CNN architecture can be improved with the use of consecutive convolutional layer with different filter. To obtain more number of learned features for the image, more convolutional layers along with filters are used and thereby spatial preservation is achieved effectively. Thus to be 
simple and sharp, in convolutional layer the feature map extracts the features that are important and essential for classification. The spatial relationship between pixel, called spatial invariance, hierarchical feature learning and scalability are preserved in convolutional layer. The convolved feature or activation maps are incorporated with ReLu function to increase non linearity of the network. Recognition of objects or images using ReLu is the transition of pixels with adjacent pixels and so images are processed even when it is rotated, tilted or shifted.

$$
f(x)= \begin{cases}0, & x<0 \\ x, & x \geq 0\end{cases}
$$

where $f(x)$ is activation function of ReLu and $x$ ranges from 0 to $\infty$. Thus CNN architecture obtain it's high performance through a supervised feature extraction, in which the learning process occurs on its own unlike other artificial neural networks. Fig 5.shows the features of the brain MRI image that are obtained from the convolutional layer of the proposed CNN Deep net.

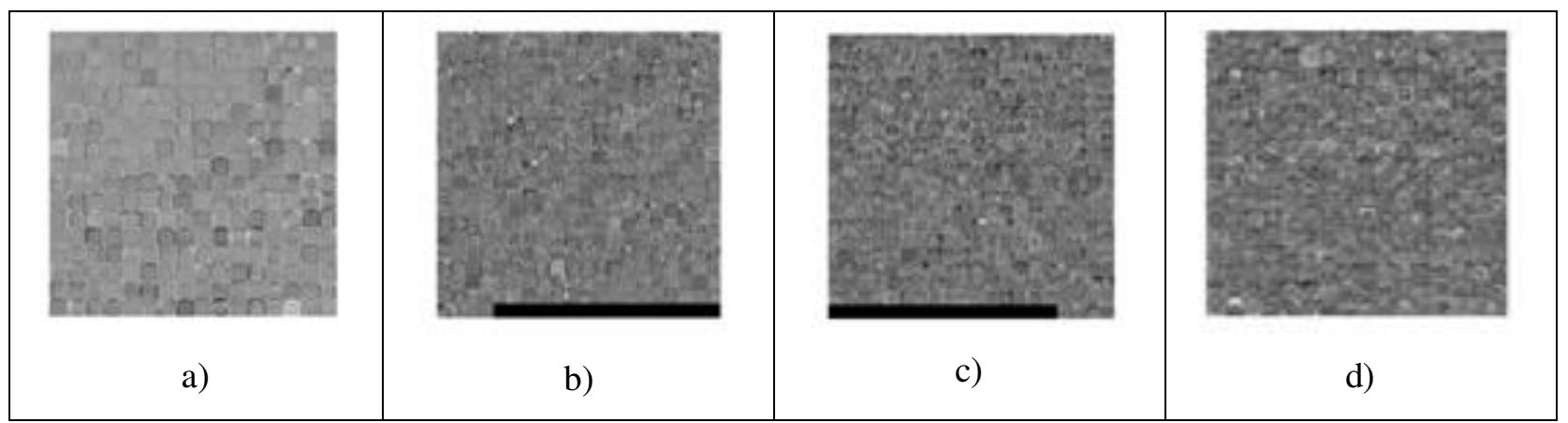

Fig .5 Features extracted from different convolutional layers of proposed CNN Deep net a) Features extracted from convolutional layer 1 with 64 kernels b). features extracted from convolutional layer 2 with 128 kernels c)features extracted from convolutional layer 3 with 256 kernels d)features extracted from convolutional layer 4 with 256 kernels.

\subsubsection{Polling layers}

The pooling of features can be done through the pooling layers which is actually a down sampling process. Here high data pixels obtained from convolved feature are minimized to a level that is suitable for further processing in neural network. Pooling layers of CNN architecture comprises of Max, Min, and Average pooling. The pooling layers, function in such a way as implied by their names and an example is illustrated in Fig. 6.The maximum value among the features is returned in Max pooling and the average of features in the region are returned in average pooling. 


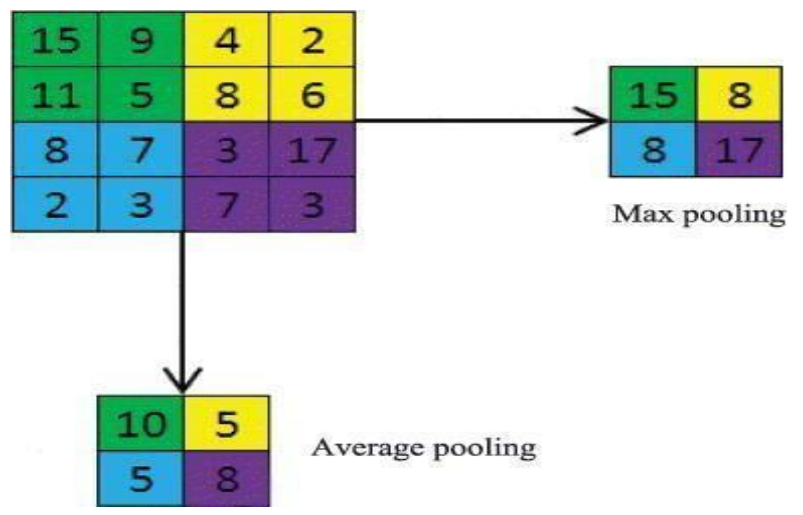

Fig. 6. Max and Average pooling

The main advantage of spatial distortion and size reduction of $75 \%$ is achieved through max pooling layer. Thus in this paper a Max pooling filter with $2 \times 2$ filter mask is used and shows better performance in classification of images. This max pooling filter is applied to the result of the convolutional layer .It is observed that the depth of feature map after applying pooling also remains same with non overlapping features. Hence the proposed method eliminates the chance of over fitting occurrence, and reduces the number of parameters to $25 \%$ when compared to the original number of parameters obtained from the input MRI brain image.

\subsubsection{Fully connected layer}

The obtained convolved features are pooled and it is required to be flatten sequentially into a single column. This flattened single sequential column achieves spatial invariance. It forms a vector of inputs that are fed to the hidden layers for classification. The flattened process is followed with fully connected layers. The flattened single vector acts as input vector and is forwarded to hidden layers through fully connection method. High level features are combined with the attributes and hence prediction of category or classes can be done even better. The fully connected layer learns all the possible non linear functions and 3D feature map is feed forwarded to produce 1D feature vector of 3D volume. In this stage, volume is deep enough with increased number of kernels, pooling layers and convolutional layers. The gradient descent approach involves only a mini batch of training set for the computation of error function in neural network. But a stochastic gradient descent approach is used to compute error function for the entire training set at a time. Hence the proposed architecture uses stochastic descent approach and thereby achieves high speed. Thus multi layer preceptor -feed forward neural network with pooling layer is proposed to perform the classification by weight adjusting operator.

\section{Proposed CNN Deep Net}

An efficient and supervised deep learning architecture is proposed for the classification of brain tumors. The proposed CNN Deep Net detects the normal and tumor brain image. As the proposed method is supervised the most important feature extraction and classification are inherently built in it and implies the process with more trainable features. In the proposed CNN Deep net the convolution process is linear and continuous. The convolutional module prepares an output by convolving preprocessed image with the 64 convolutional kernel filter. The kernel filter of $3 \times 3$ with single stride is involved in the process. This convolution ends up with $256 \times 256 \times 64$ feature maps which are 
stacked along their depths to create an output volume .The convolutional layer results in different features that are dictated by kernel using convolution. And moreover no padding is required is the proposed architecture. The framework of the proposed method is shown in Fig 7.

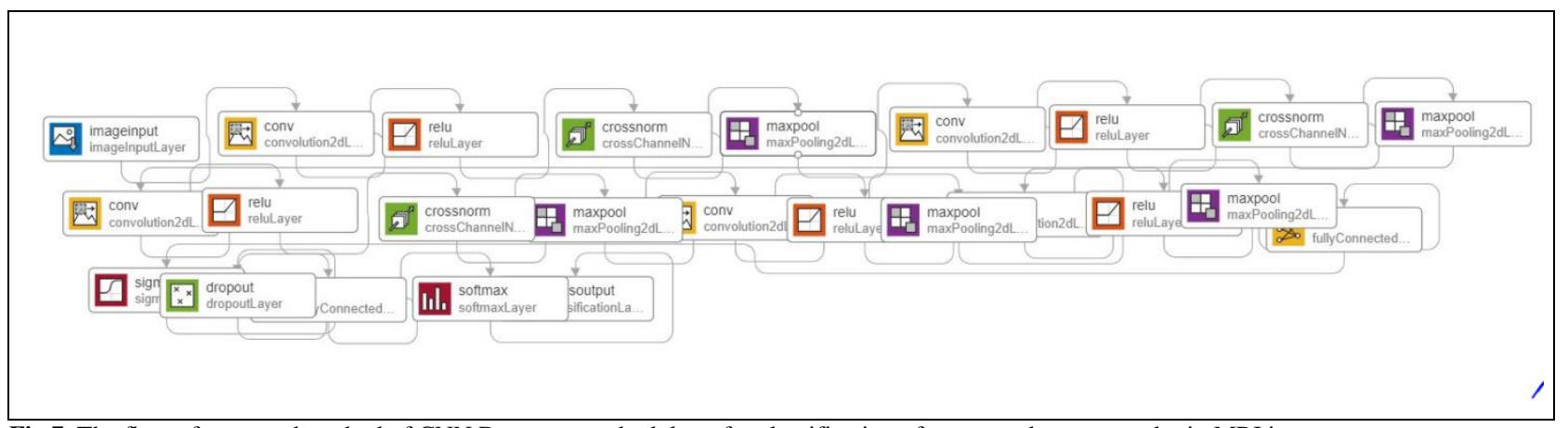

Fig 7. The flow of proposed method of CNN Deep net methodology for classification of tumor and non tumor brain MRI images

In this paper, large number of features and parameters are obtained from the flattened layer's single column vector. The proposed CNN Deep net consists of two fully connected hidden layer for two classes and an output classification layer with softmax activation. The neuron in output layer when stated as 0 , represents the non-tumor case and represents the tumor case when it stated 1.

Table 2 shows the number of layers with its output shape and learnable parameters of the proposed CNN Deep net. This analysis report is obtained using deep learning network analysis tool .The analysis of deep learning network is simulated from MATLAB R2020b.The activations size, number of extracted features and learnable parameters with weights and bias are tabulated.The total learnable parameters of the proposed CNN Deep net are 5, 66, 24,136.

\begin{tabular}{|c|c|c|c|c|}
\hline Layers & Layer size & Output size & Learnables & $\begin{array}{l}\text { Parameters/ } \\
\text { features }\end{array}$ \\
\hline Image input layer & $256 \times 256 \times 3$ & & - & 0 \\
\hline Conv2D1 with ReLU & $64 \times 3 \times 3$ & $254 \times 254 \times 3$ & 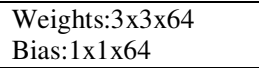 & 1792 \\
\hline Maxpool1 & $2 \times 2$ & $127 \times 127 \times 3$ & - & \\
\hline Conv2D2 with ReLU & $128 \times 3 \times 3$ & $125 \times 125 \times 3$ & 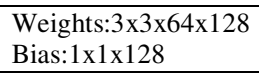 & 13856 \\
\hline Maxpool2 & $2 \times 2$ & $62 \times 62 \times 3$ & - & - \\
\hline Conv2D3 with ReLU & $256 \times 3 \times 3$ & $60 \times 60 \times 3$ & $\begin{array}{l}\text { Weights: } 3 \times 3 \times 256 \\
\text { Bias:1x1x256 }\end{array}$ & 295168 \\
\hline Maxpool3 & $2 \times 2$ & $30 \times 30 \times 3$ & - & - \\
\hline Conv2D4 with ReLU & $256 \times 3 \times 3$ & $28 \times 28 \times 3$ & $\begin{array}{l}\text { Weights: } 3 \times 3 \times 256 \\
\text { Bias:1x1x256 }\end{array}$ & 590080 \\
\hline Maxpool4 & $2 \times 2$ & $14 \times 14 \times 3$ & & \\
\hline Conv2D5 with ReLU & $256 \times 3 \times 3$ & $12 \times 12 \times 3$ & $\begin{array}{l}\text { Weights: } 3 \times 3 \times 256 \\
\text { Bias:1x1x512 }\end{array}$ & 1179904 \\
\hline Maxpool5 & $2 \times 2$ & $6 \times 6 \times 3$ & & \\
\hline Fully connected Layer & - & 9216 & $\begin{array}{l}\text { Weights:2x26382848 } \\
\text { Bias: } 2 \times 1\end{array}$ & 37752832 \\
\hline
\end{tabular}




\begin{tabular}{|l|l|l|l|l|}
\hline Fully connected Layer & - & 4096 & & 16781312 \\
\hline Softmax & 2 & 9192 & - & - \\
\hline $\begin{array}{l}\text { Classification Output } \\
\text { layer }\end{array}$ & 1 & - & - & - \\
\hline
\end{tabular}

Table 2: Parameter Summary of the proposed CNN Deep net

\subsection{Segmentation}

The classified meningioma brain tumor image is segmented to obtain dilated and eroded images by global opening and closing functions respectively. These two images are subtracted to obtain threshold image. In order to improve the segmentation process, an area morphological function is utilized in threshold image to eliminate the missed pixels.Here, an important process of image analysis called labeling is carried through connected component method .The connected component method forms a group based on its pixel connectivity. The labeling is applied to the threshold image to segregate the tumor regions of brain from normal regions. The MRI image is scanned pixel pixel to group the same intensity values and 8 pt-connectivity is assumed for analysis. Experimental results of segmentation show better performance with sharp boundary detections of tumor pixels. The boundary detection of the proposed method shows high effectiveness in comparison to conventional region growing algorithms. The segmented tumor region using proposed method is shown in Fig.8.

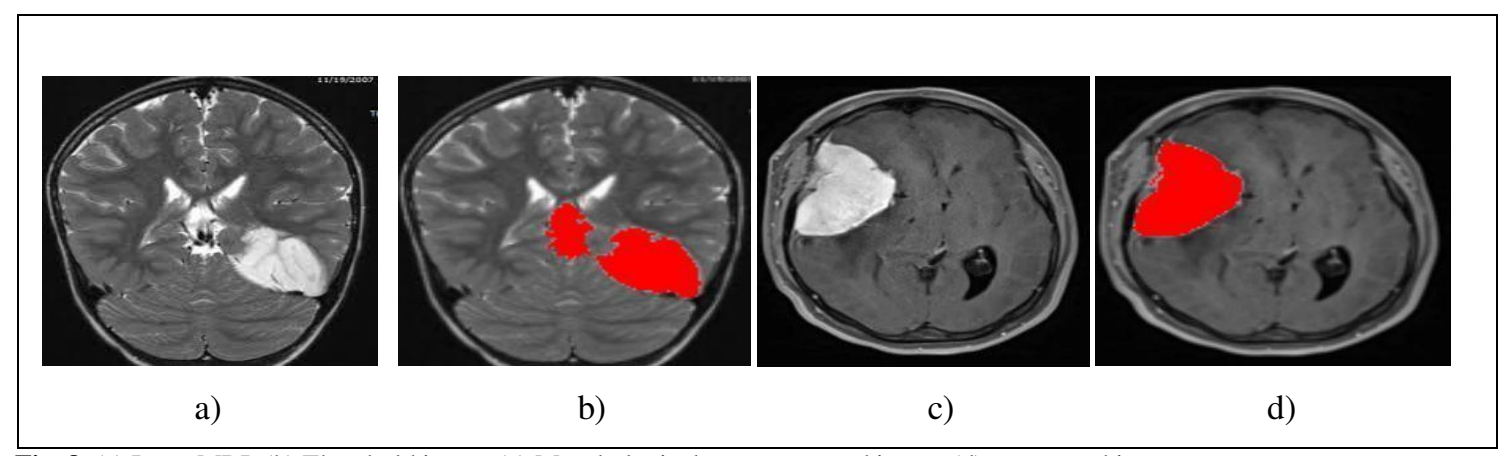

Fig. 8. (a) Input MRI (b) Threshold image (c) Morphological area processed image (d) segmented image

\subsection{Multi preceptor CNN based Diagnosis system:}

The CNN architecture represents low grade (benign) and high grade (malignant) tumor using a methodology of extracting the features of segmented tumor regions. The grade classification is performed by grey level cooccurrence matrix (GLCM).The features of tumor regions obtained from GLCM feature extraction forms a vector matrix. Grade classification is done on this vector matrix through the CNN classification layer. The proposed diagnosis system for grading uses single neuron at the output layer .The classification result of 0 or 1 is obtained from $\mathrm{CNN}$ which indicates low grade and high grade case respectively. Fig. 9 illustrates the diagnosis process. 


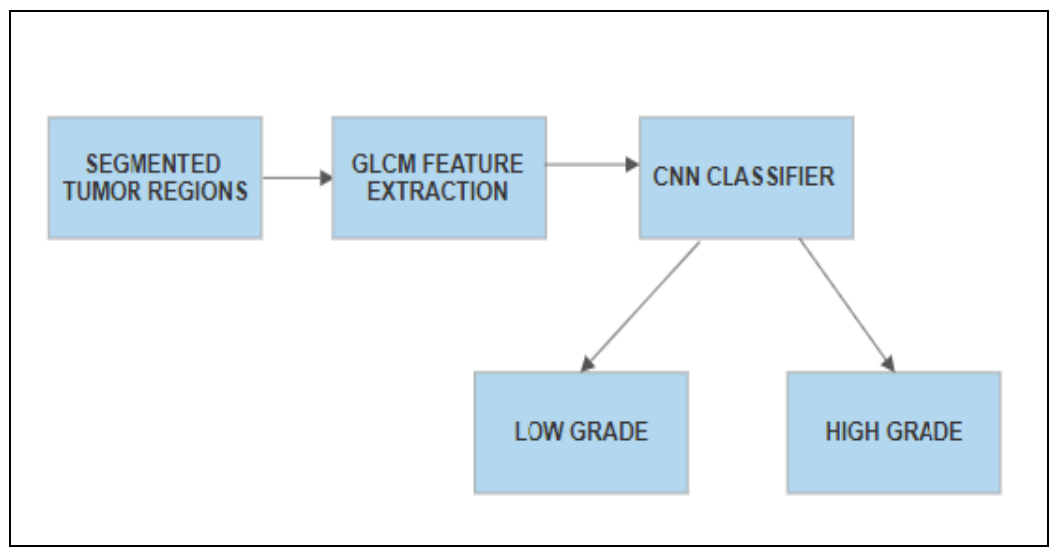

Fig.9.Proposed brain tumor diagnosis system

\subsubsection{GLCM-CNN classifier:}

Texture properties of segmented tumor region are computed using GLCM features. These GLCM features are spatial features obtained from co-occurrence matrix with 90 degree from preprocessed brain image. The various GLCM features suited for the proposed methodology are contrast, entropy, mean and homogeneity. In this proposed diagnosis, 260 brain MRI images with 156 low grade cases (benign) and 104 high grade cases (malignant) are trained. A total number of features extracted from images are 1560 as each image computes 6 features. These obtained features in vector are applied to fully connected classification layer of CNN classifier to classify low grade and high grade tumor. The GLCM features used for the proposed approach are stated below in the equations (8), (9), (10) and (11).

$$
\begin{aligned}
& \text { Contrast }=\sum_{i=0}^{P-1} \sum_{j=0}^{Q-1}(i-j)^{2} * P(i, j) \\
& \text { Entropy }(E N)=\sum_{i=0}^{P-1} \sum_{j=0}^{Q-1} P(i, j) * \log P(i, j) \\
& \text { Mean }=\mu_{i}=\sum_{i=0}^{P-1} i * P(i, j) \\
& \text { Homogeneity }(H)=\sum_{i=0}^{P-1} \sum_{j=0}^{Q-1} \frac{1}{1+(i-j)^{2}} * P(i, j)
\end{aligned}
$$

where, $P(i, j)$ is the GLCM matrix constructed at the orientation of 90 degree, the rows and columns of the GLCM matrix are represented as $\mathrm{i}$ and $\mathrm{j}$. The number of rows and columns in GLCM matrix are represented by $\mathrm{P}$ and $\mathrm{Q}$, respectively.

Fig. 10(a) and Fig. 10(b) shows the proposed GLCM-CNN classifier results for low grade brain MRI images and high grade brain images. 


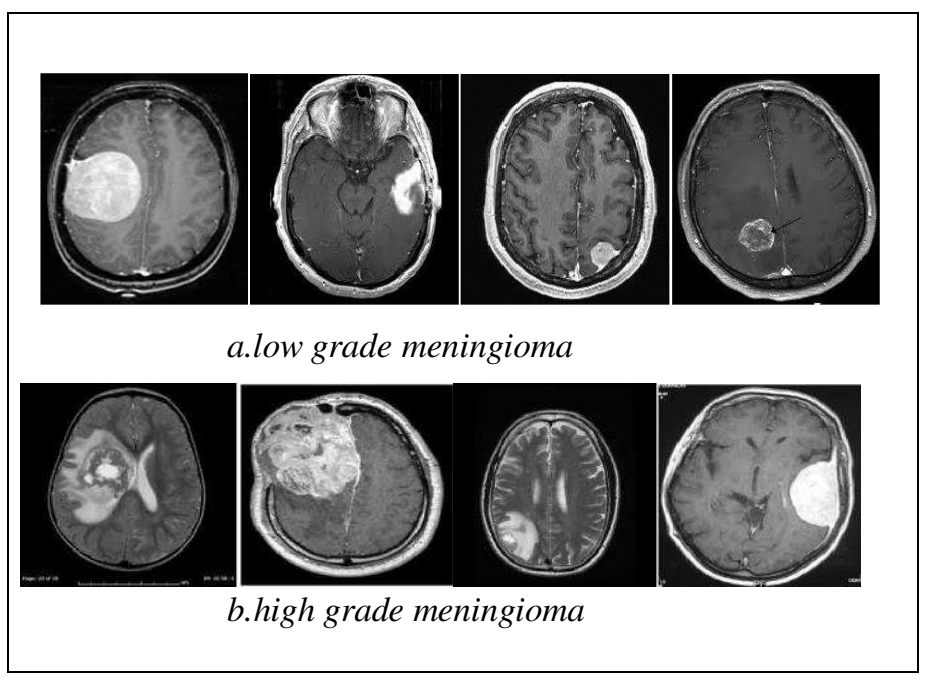

Fig 10.Classification results of the proposed CNN Deep net a) low grade tumor b) high grade tumor

\section{Results and Discussion}

In this article, the proposed CNN Deep net detection and classification methodology is applied on the set of brain MR images in the BRATS, an open access dataset. From the dataset, 600 brain images of $512 * 512$ pixels are used in this article which includes 340 normal brain images and 260 tumor affected brain images. MATLAB R2020 b is used as simulation tool for detection, classification and segmentation of tumor region with Intel core i5 processor with $2.30 \mathrm{GHz}$ and 16GB RAM memory.

Table 3 shows the performance metrics of proposed CNN Deep net classifier on BRATS brain MR image datasets. The validation accuracy, validation loss and training loss with different epochs are obtained from simulation results and the average accuracy of proposed Deep net is tabulated as shown.

\begin{tabular}{|c|c|c|c|c|c|}
\hline Epoch & Training Loss & Val.Loss & Val.Acc & Time & Results (\%) \\
\hline 1 & 0.6703 & 0.4316 & 0.8696 & 19 & 62.6 \\
\hline 2 & 0.3982 & 0.1319 & 0.9565 & 29 & 82.39 \\
\hline 3 & 0.1346 & 0.0223 & 1.000 & 27 & 90.43 \\
\hline 4 & 0.0486 & 0.0858 & 0.9565 & 28 & 92.26 \\
\hline 5 & 0.0469 & 0.0204 & 0.9582 & 25 & 95.26 \\
\hline 6 & 0.0162 & $1.18 \mathrm{e}-04$ & 0.9652 & 27 & 96.5 \\
\hline 7 & 0.0115 & $7.044 \mathrm{e}-04$ & 0.9693 & 26 & 96.9 \\
\hline 8 & 0.0066 & $2.298 \mathrm{e}-05$ & 0.9791 & 28 & 97.5 \\
\hline 9 & $8.54 \mathrm{e}-04$ & $9.147 \mathrm{e}-05$ & 0.9822 & 32 & 98.7 \\
\hline 10 & $3.45 \mathrm{e}-04$ & $4.24 \mathrm{e}-05$ & 0.9941 & 47 & 99.5 \\
\hline
\end{tabular}

Table 3: Performance metrics of proposed CNN Deep net for classification of normal and tumor brain image

The performance of the proposed CNN Deep net depend on its accuracy and loss values against the epoch .Thus a accuracy and loss plots are plotted against the epoch for training and test datasets. Fig 11 shows the performance analysis of proposed CNN Deep net for classification of brain tumor images graphically. The parameters are hypertuned for the improved performance of proposed Deep net .A Mini batch of 64,batch 
regularization,normalization and 50\% dropout with 0.01 learning rate are performed as hypertuning.

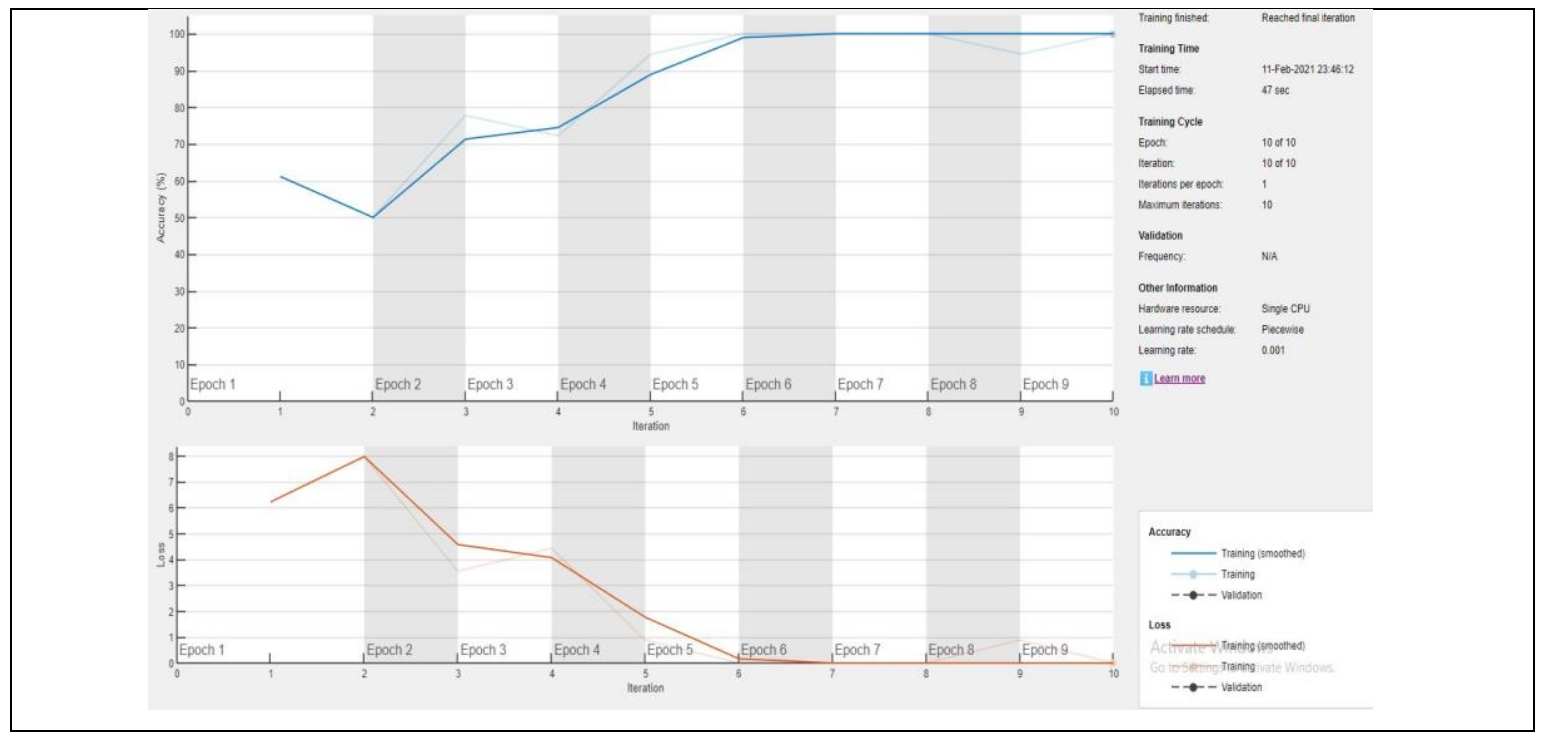

Fig.11.Performance analysis of proposed CNN Deep net for classification of brain tumors images with different epoch

\subsection{Performance evaluation metrics}

Both subjective and objective comparison of classification and segmentation are well explained in terms of sensitivity, specificity, accuracy, precision, F score, DSI etc. Analysis of the proposed CNN Deep net is performed by means of classification rate which is the ratio of number of correctly classified objects to the total objects involved in the method.

In this proposed method the classification rate of normal image and affected tumor image is $99.5 \%$ when 340 normal MRI images and 206 affected tumor MRI images are considered for classification. It is thus found that an average of 99.5\% classification rate and validation accuracy $99.4 \%$ is achieved through the proposed method.

Further, the following performance evaluation metrics are considered for the proposed CNN Deep net brain tumor detection system. The correlation between the correctly classified pixels are well stated by sensitivity and specificity where accuracy defines the rate of correctly detected pixels in segmented brain image. The tumor free healthy pixels are detected and stated through Precision. Disc Similarity Index is used to analysis the correctly detected pixels that are similar to each other when compared to ground truth image. All these parameters are measured in percentage and vary between 0 and 100 .

- $\quad$ Sensitivity $(\operatorname{Sen})=T P /(T P+F N))$

- $\operatorname{Specificity~}(S p=T N /(T N+F P))$

- $\operatorname{Accuracy}(A C C)=(T P+T N) /(T P+F N+T N+F P))$

- $\operatorname{Precision}(\operatorname{Pr})=T P /(T P+F P)$

- $F-$ score $=2 * \operatorname{Sen} * \operatorname{Pr} /(\operatorname{Pr}+\operatorname{Sen})$

- $\quad$ Dice Similarity Index $(D S I)=2 * T P /(T P+T N+F P+F N)$

The evaluation metrics for the performance analysis of the proposed method is stated through a confusion matrix 
which has the value of TP and TN, providing correctly detected tumor and correctly detected non tumor pixels as well as FP and FN to provide non-correctly detected tumor and non-correctly detected non tumor pixels .

Table 4 is illustrated from confusion matrix and listed as sensitivity (Sen),Specificity(Sp),Accuracy(Acc),Precision(Pr),F-score, Dice Similarity Index (DSI).

\begin{tabular}{|c|c|}
\hline Metrics & Results (\%) \\
\hline Sen & 97.2 \\
\hline Sp & 98.6 \\
\hline Acc & 99.4 \\
\hline Pr & 97.6 \\
\hline F-score & 98.1 \\
\hline DSI & 98.7 \\
\hline
\end{tabular}

Table.4 Performance metrics of the proposed CNN Deep net classifier

From Table 5, the meningioma brain tumor detection using CNN Deep Net architecture provides higher classification rate while compared with the brain tumor detection system using conventional CNN architecture.

\begin{tabular}{|c|c|c|}
\hline CNN architecture types & Pooling layer & Classification rate (\%) \\
\hline LeNET CNN architecture (Bakhshi, et al. & Average Pooling layer & 92.1 \\
\cline { 2 - 3 } 2019$)$ & Max Pooling layer & 94.7 \\
\hline \multirow{2}{*}{ CNN Deep Net architecture } & Average Pooling layer & 97.6 \\
\cline { 2 - 3 } & Max Pooling layer & 99.5 \\
\hline
\end{tabular}

Table 5 Classification rate analysis with respect to different CNN architectures and pooling layers

Table 6 is the comparisons of proposed simulation results with other conventional methods on same dataset images. It is inferred from these comparisons, the proposed system using CNN Deep net classification approach yields high simulation values when compared with other conventional methods on same dataset brain MRI images.

\begin{tabular}{|c|c|c|c|c|c|c|c|}
\hline Classifiers & Approach & Classes & Preprocessing & $\begin{array}{c}\text { Feature } \\
\text { extraction }\end{array}$ & Acc(\%) & $\begin{array}{c}\text { Sen(\%) } \\
\text { Spe } \\
\text { (\%) }\end{array}$ \\
\hline SVM & ML & 2 & YES & GLCM & 96.9 & 94.6 & 96.1 \\
\hline CANFIS & ML & 2 & YES & $\begin{array}{c}\text { DWT \& } \\
\text { GLCM }\end{array}$ & 98.8 & 96.5 & 97.7 \\
\hline $\begin{array}{c}\text { Aripathi et al. } \\
\text { (2019) }\end{array}$ & Decision tree & 2 & YES & PCA \& GLCM & 92.8 & 94.5 & 94.1 \\
\hline $\begin{array}{c}\text { CNN } \\
\text { Milica M. Badža } \\
\text { et al. (2020) }\end{array}$ & CNN & 2 & YES & Convolutional & 96.5 & 96.1 & 92.1 \\
\hline $\begin{array}{c}\text { Rajagopal } \\
\text { (2019) }\end{array}$ & $\begin{array}{c}\text { Random forest } \\
\text { classifier }\end{array}$ & 2 & layer & & 96.7 & 97.7 \\
\hline
\end{tabular}




\begin{tabular}{|c|c|c|c|c|c|c|c|}
\hline $\begin{array}{c}\text { Josephine et al. } \\
\text { 2018) }\end{array}$ & $\begin{array}{c}\text { Genetic } \\
\text { Algorithm }\end{array}$ & 2 & YES & GA & 91.0 & 90.6 & 91.7 \\
\hline $\begin{array}{c}\text { Rohit et al. } \\
(2013)\end{array}$ & $\begin{array}{c}\text { Fuzzy C-mean } \\
\text { algorithm }\end{array}$ & 2 & YES & Fuzzy layer & 90.6 & 91.7 & 92.5 \\
\hline $\begin{array}{c}\text { Proposed CNN } \\
\text { Deep Net }\end{array}$ & DL & 2 & YES & $\begin{array}{c}\text { Convolutional } \\
\text { layer }\end{array}$ & 99.4 & 97.2 & 98.6 \\
\hline
\end{tabular}

*ML-machine learning , *DL-Deep learning

Table 6. Comparision of the proposed CNN classifier with machine learning classifiers

On the basis of centroid index on segmented region the morphological parameters are calculated and their values are tabulated in Table 7 with circumference $(\mathrm{C})$, perimeter $(\mathrm{P})$, area(A) and eccentricity(Ecc) .

\begin{tabular}{|c|c|}
\hline Parameters & Value \\
\hline $\mathrm{C}(\mu \mathrm{m})$ & 127.66 \\
\hline $\mathrm{P}(\mu \mathrm{m})$ & 67.19 \\
\hline $\mathrm{A}(\mu \mathrm{m} 2)$ & 156.47 \\
\hline $\mathrm{Ecc}$ & 0.864 \\
\hline
\end{tabular}

Table 7: Analysis of Segmented tumor regions

In this paper, 156 low grade cases and 104 high grade cases with a total of 260 tumor affected MRI brain images are used for diagnosis. The features extracted from segmented region are tabulated for both low grade and high grade cases below. Table 8 shows the GLCM features of low grade and high grade tumor brain MRI images.

\begin{tabular}{|c|c|c|}
\hline GLCM Features & Low grade & High grade \\
\hline Contrast & 0.256 & 0.335 \\
\hline Entropy & 0.655 & 2.012 \\
\hline Mean & 8.661 & 11.321 \\
\hline Homogeneity & 0.920 & 0.894 \\
\hline
\end{tabular}

Table 8: GLCM features of low grade and high grade tumor brain MRI images

A diagnosis rate of $98.6 \%$ is obtained, as the proposed system classifies the low grade cases correctly with the ratio of 155:156 and similarly produces $98.07 \%$ of diagnosis rate while classifying high grade cases correctly. Thus the proposed method has obtained $98.3 \%$ diagnosis average rate. The diagnosis result of the proposed method is tabulated in Table 9.

\begin{tabular}{|c|c|c|c|}
\hline Grades & Images & $\begin{array}{c}\text { Classified } \\
\text { images }\end{array}$ & $\begin{array}{c}\text { Rate of diagnosis } \\
(\boldsymbol{\%})\end{array}$ \\
\hline Low grade & 156 & 155 & 98.6 \\
\hline High grade & 104 & 103 & 98.0 \\
\hline
\end{tabular}

Table 9: Analysis of Diagnosis system using proposed method 


\subsection{Discussion}

It is observed that the metrics sensitivity, specificity, accuracy, precision and F-score of the proposed CNN Deep Net works better when compared with the conventional machine learning methods. Table 10 shows the comparison of the proposed CNN Deep Net architecture for meningioma classification and diagnosis with the pre-trained CNN architectures.

\begin{tabular}{|c|c|c|c|c|}
\hline Classifier & $\begin{array}{c}\text { Input } \\
\text { image size }\end{array}$ & $\begin{array}{c}\text { No. of } \\
\text { Layers }\end{array}$ & $\begin{array}{c}\text { Hyper } \\
\text { tuning }\end{array}$ & Acc (\%) \\
\hline Proposed CNN Deep Net & $256 \times 256 \times 3$ & 7 & $\checkmark$ & 99.4 \\
\hline ALEXNET & $227 \times 227 \times 3$ & 8 & $\checkmark$ & 98.14 \\
\hline VGG 19 & $224 \times 224 \times 3$ & 26 & $\checkmark$ & 97.97 \\
\hline GOOGLENET & $299 \times 299 \times 3$ & 50 & $\checkmark$ & 95.69 \\
\hline
\end{tabular}

Table 10. Comparison of the proposed CNN Deep net with pre-trained CNN networks

\section{Conclusion}

In this paper, detection of meningioma brain tumor and classification by CNN Deep net is proposed. The CNN Deep net architecture is designed with five convolutional layer with ReLU activations, Max pooling layers and multi neuron feed forward neural network to obtain deep features from input brain MRI images. The proposed methodology uses multilayer perceptron architecture for detection and classification of brain tumor with grade classification. Global threshold segmentation approach is used for segmenting the detected tumor affected region where both dilation and erosion are used for locating the tumor regions. Further, a novel diagnosis system using GLCM CNN classifier is proposed and achieves a high classification rate and accuracy. Open access dataset BRATS is used for the analysis and evaluation of the proposed method. Thus the proposed CNN Deep net architecture has achieved higher classification rate of $99.5 \%$ with better specificity ( $98.6 \%)$ and sensitivity $(97.2 \%)$. The Deep net achieves a segmentation accuracy of $99.4 \%$ where the diagnosis of segmented tumor regions achieves greater diagnosis rate of $98.3 \%$.

\section{Conflict of interest}

The authors declare that they have no conflict of interest.

\section{Acknowledgment}

The authors would like to thank their family and colleagues for their constant help and support throughout the study to obtain the results.

\section{Ethical Approval}

Not applicable.

\section{Consent to Participate}

The authors declare that they have consent to participate in journal submission. 


\section{Consent to publish}

The authors declare that they have consent to publish article in BIOR journal.

\section{Authors contribution}

All the authors have equal contribution in this research work.

\section{Funding}

The authors declare that they have no funding.

\section{Availability of data and materials}

The brain images used in this article are available at https://www.smir.ch/BRATS. This dataset is open access and license free.

\section{References}

[1] Bandhyopadhyay, S.K., Paul, T.U. Automatic segmentation of brain tumour from multiple images of brain MRI, International Journal of Application or Innovation in Engineering \& Management (IJAIEM), 2 (2013) 240-248.

[2] BRATS database, (2015) Available at: 〈https://www.smir.ch/BRATS/Start2015>

[3] El-Melegy, M.T., Mokhtar, H.M. Tumor segmentation in brain MRI using a fuzzy approach with class center priors, EURASIP Journal on Image and Video Processing 2014 (2014). doi: 10.1186/1687-5281-2014-21.

[4] Funmilola, A. Oke, T Adedeji, M. Alade, E.A. Adewusi, Fuzzy K-C-means clustering algorithm for medical image segmentation, J Informat Eng Appl 2 (2012) 21-32. https://pdfs.semanticscholar.org/da58/e39c29c44560426c2fb00b0f8dbeec5c0dd6.pdf.

[5] C.H. Sudre, Li W., T. Vercauteren, S. Ourselin, M.J. CardosoGeneralised Dice overlap as a deep learning loss function for highly unbalanced segmentations Proceedings of MICCAI Workshop on Deep Learning in Medical Image Analysis (DLMIA) (2017)

[6] Abdelmajid Bousselham, Omar Bouattane, Mohamed Youssfi, and Abdelhadi Raihani. Towards Reinforced Brain Tumor Segmentation on MRI Images Based on Temperature Changes on Pathologic Area. International Journal of Biomedical Imaging, 2019, 1758948, 1-18, (2019).

[7] Alqazzaz S, Sun X, Yang X, et al. "Automated brain tumor segmentation on multi-modal MR image using SegNet.. Comp. Visual Media, 5, 209-219, (2019)

[8] Shen D., Wu G., Suk H.-I.Deep learning in medical image analysis Ann. Rev. Biomed. Eng. (19) (2017), pp. 221-248

[9] Li Sun ,Songtao zhang,Hang zen ,"Brain tumor segmentation and survival prediction using multimodal MRI scans with deep learning “,frontier neuroscience, $13,819(2019)$

[10] Guo L, Zhao L, Wu Y, Li Y, Xu G, and Yan Q. Tumor detection in MR images using one-class immune feature weighted SVMs. IEEE Transactions on Magnetics, 47, 10, 3849-3852, (2011).

[11] Kai Roman Laukamp .et.al" Fully automated detection and segmentation of meningiomas using deep learning on routine multiparametric MRI,” European Radiology ,29,124-132(2019).

[12] Sajid Iqbal et.al "Deep learning model integrating features and novel classifiers fusion for brain tumor segmentation ",microscopic research and techniques -Wiley,1302-1315(2019)

[13] Islam A, Reza SMS, and Iftekharuddin KM, "Multifractal texture estimation for detection and segmentation of brain tumors. IEEE Trans. Biomed. Eng., 60, 11, 3204-3215, (2013).

[14] Josephine S. Brain Tumor MRI Image Detection and Segmentation Using Genetic Algorithm. International Journal of Computer Sciences and Engineering, 6, 2, (2018).

[15] Kong Y, Deng Y, and Dai Q. Discriminative clustering and feature selection for brain MRI segmentation. IEEE Signal Processing Letters, $22,5,573-577,(2015)$.

[16] Guotai Wang, Wenqi Li, S'ebastien Ourselin, and Tom Vercauteren,” Automatic Brain Tumor Segmentation using Cascaded Anisotropic Convolutional Neural Networks,"arXiv:1709 (2017)

[17] Menze B, Reyes, M, van Leemput K. The multimodal brain tumor image segmentation benchmark (BRATS). IEEE Transactions on Medical Imaging, 34, 10, 1993-2024, (2015).

[18] Pereira S, Pinto A, Alves V, Silva CA. Brain tumor segmentation using convolutional neural networks in MRI images. IEEE Transactions on Medical Imaging, 35, 5, 1240-1251, (2016).

[19] Rao CH, Naganjaneyulu PV, and Prasad KS. Brain Tumor Detection and Segmentation Using Conditional Random Field. IEEE 7th International Advance Computing Conference (IACC), Hyderabad, 807-810, (2017).

[20] Muhammad Sajjada, Salman Khanb, Khan Muhammad,Wanqing Wu, Amin Ullah, Sung Wook Baik ," Multi-grade brain tumor classification using deep CNN with extensive data augmentation", 1877-7503, Journal of Computational Science-Elsevier,30,174182(2019)

[21] Rohit M, Kabade S, Gaikwad MS. Segmentation of brain tumour and its area calculation in brain MRI images using K-mean clustering and Fuzzy C-mean algorithm. Int J Comput Sci Eng Technol (IJCSET), 4, 5, 524-531, (2013).

[22] Javaria Amin, Muhammad Sharif , Mudassar Raza , Tanzila Saba , Muhamma Almas Anjum," Brain tumor detection using statistical and machine learning method," Computer Methods and Programs in Biomedicine-Elsevier ,177,69-79(2019).

[23] Shelhamer E, Long, J, Darrell, T. Fully convolutional networks for semantic segmentation. IEEE Transactions on Pattern Analysis and Machine Intelligence, 39, 4, 640-651, (2017).

[24] Sonali Bansal, Shubpreet Kaur, Navdeep Kaur. Enhancement in Brain Image Segmentation using Swarm Ant Lion Algorithm. International Journal of Innovative Technology and Exploring Engineering (IJITEE), 8, 10, (2019). 
[25] P.G.Akila,Dr.K.Batri,Survey on Different Preprocessing Methods of Enhancement and Segmentation in Medical Images Analysis, Jour of Adv Research in Dynamical \& Control Systems, Vol. 10, 14-Special Issue, 2018

[26] Xia Z, Ma X, Shen Z, Sun X, Xiong NN, and Jeon B. Secure Image LBP Feature Extraction in Cloud-Based Smart Campus. IEEE Access, 6, 30392-30401, (2018).

[27] P.G.Akila,Dr.K.Batri, Detection and diagnosis of brain tumors using deep learning convolutional neural networks,(2020) https://doi.org/10.1002/ima.22532

[28] Urban, G., Bendszus, M., Hamprecht, F.A., Kleesiek, J. Multi-modal brain tumor segmentation using deep convolutional neural networks, MICCAI Multimodal Brain Tumor Segmentation Challenge (BRATS), (2014) 1-5. https://hci.iwr.uni-heidelberg.de/node/4859.

[29] Wang, G., Xu, J., Dong, Q., Pan, Z. Active contour model coupling with higher order diffusion for medical image segmentation, International Journal of Biomedical Imaging 2014 (2014) 1-8. http://dx.doi.org/10.1155/2014/237648

[30] Amin Kabir Anaraki ,Moosa Ayati ,Foad Kazemi, Magnetic resonance imaging-based brain tumor grades classification and grading via convolutional neural networks and genetic algorithms,(2019),volume 39,63-74, https://doi.org/10.1016/j.bbe.2018.10.004.

[31] Mohammad Havaei, Axel Davy, David, WF, Antoine Biard, Aaron Courville \& Yoshua Bengio 2017, 'Brain tumor segmentation with Deep Neural Networks', Medical Image Analysis, vol. 35, pp. 18 -31.

[32] Konstantinos, KK, Christian Ledig, Virginia, FJ, Joanna, PS, Andrew, DK, David, KM. 2017, 'Efficient multi scale 3D CNN with fully connected CRF for accurate brain lesion segmentation', Medical Image Analysis, vol. 36, pp. 61-78.

[33] Rajagopal R, "Glioma brain tumor detection and segmentation using weighting random forest classifier with optimized ant colony features", International Journal of imaging systems and technology, Vol.29, No.3, pp. 353-359, 2019.

[34] Khawaldeh, Saed, et al. Noninvasive grading of glioma tumor using magnetic resonance imaging with convolutional neural networks. Applied Sciences, Vol 8, 2017.

[35] Sheela, C.J.J., Suganthi, G. Morphological edge detection and brain tumor segmentation in Magnetic Resonance (MR) images based on region growing and performance evaluation of modified Fuzzy C-Means (FCM) algorithm. Multimed Tools Appl 79, 17483-17496, 2020. 


\section{Figures}
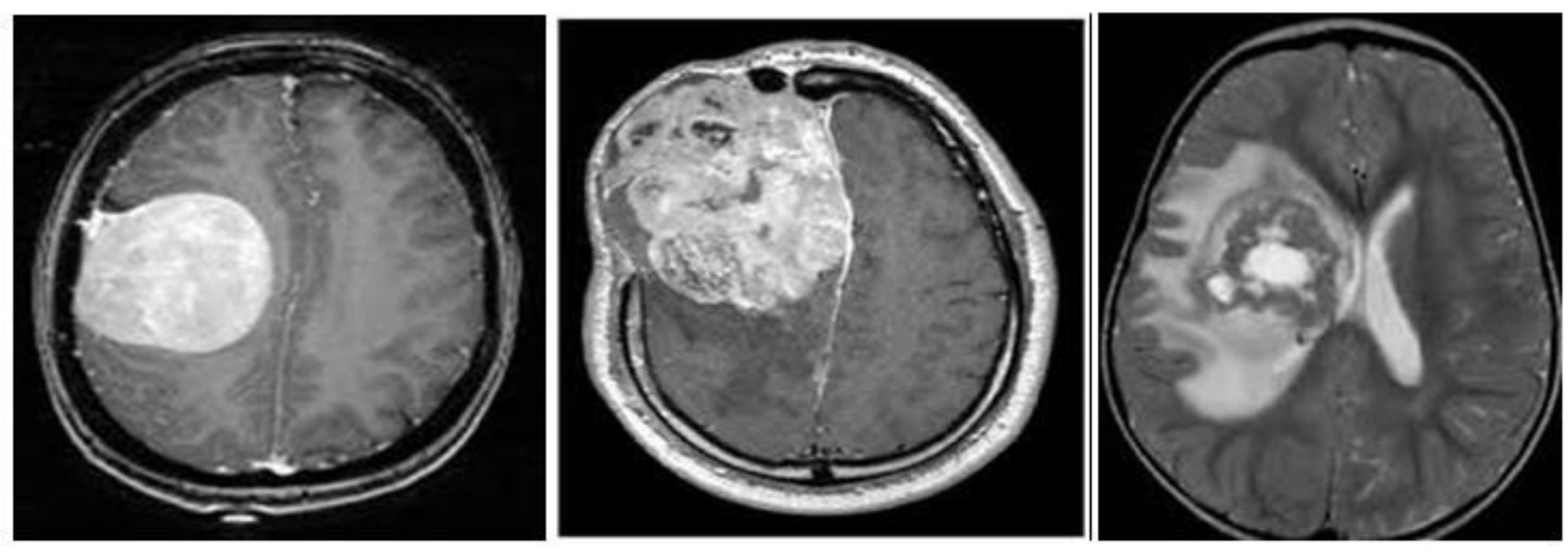

Figure 1

a) Brain Tumor MR image b) benign c) malignant meningioma

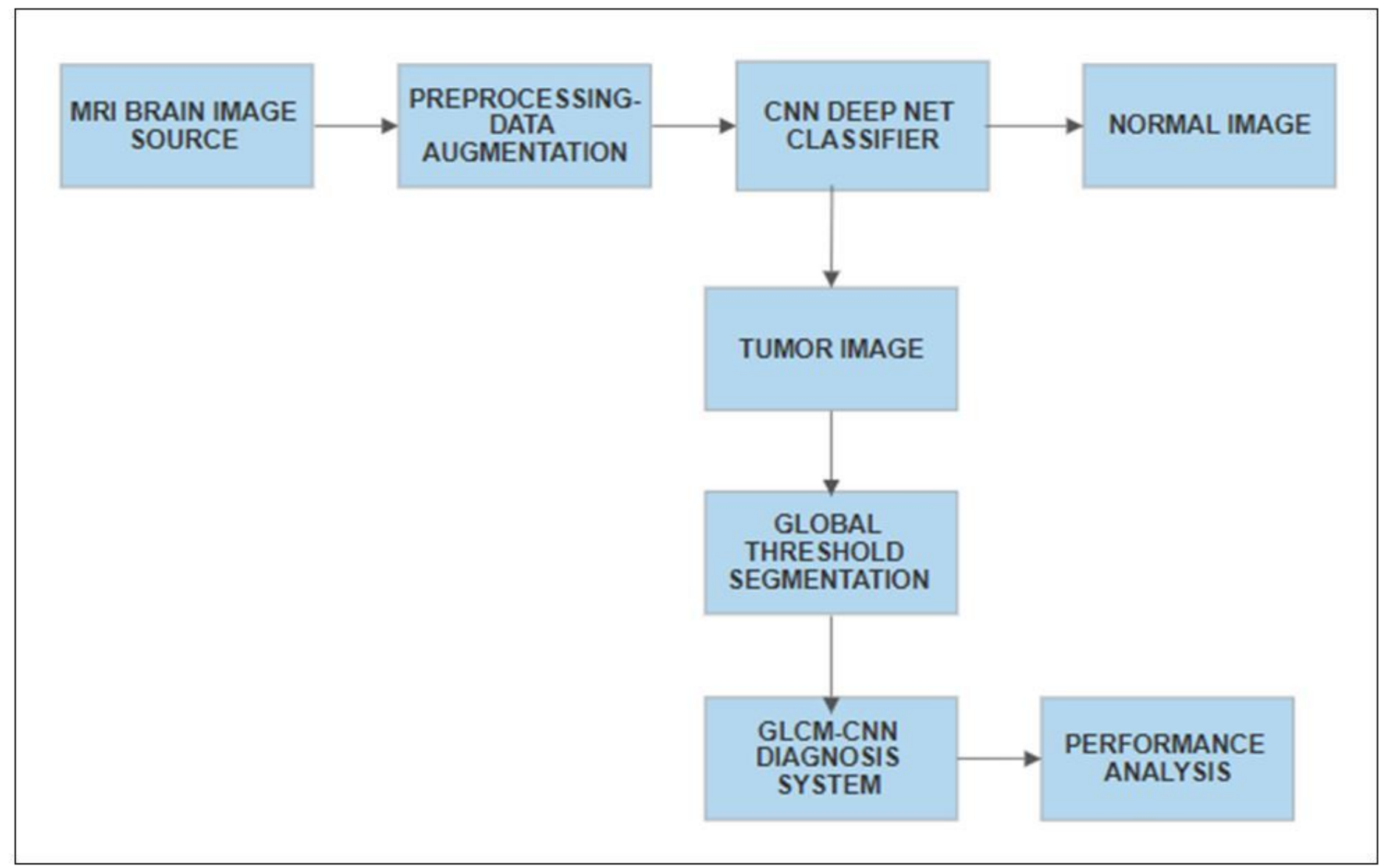

Figure 2 
Proposed brain tumor classification using CNN architecture

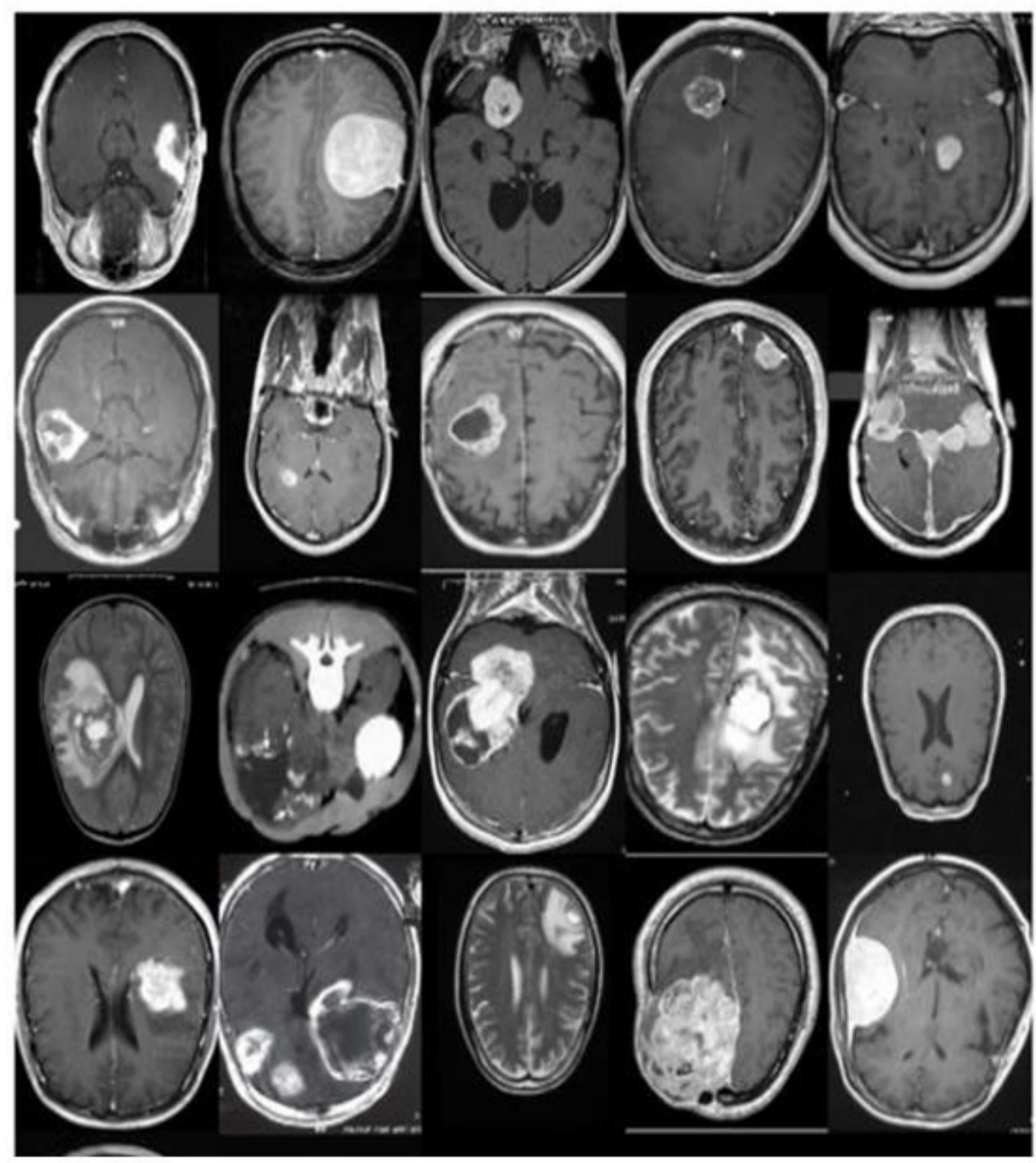

Figure 3

Data Augmented outputs of brain MRI image

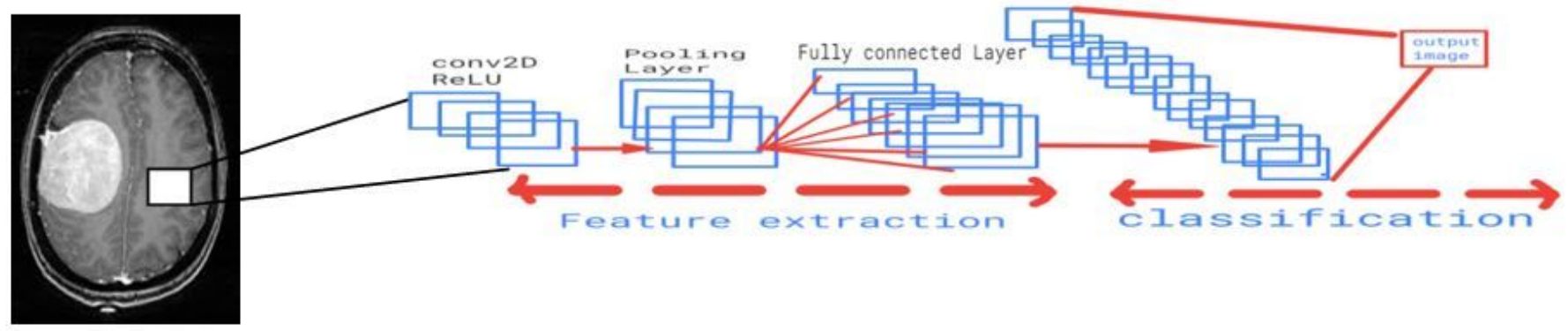


Figure 4

Proposed CNN classifier architecture

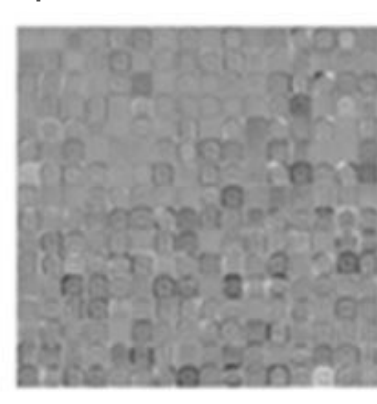

a)

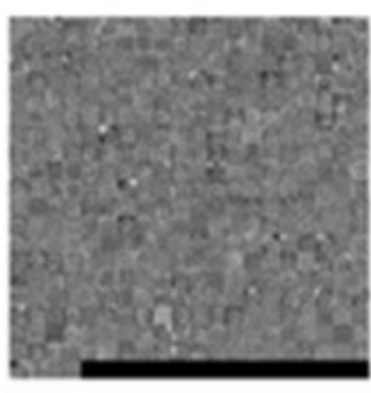

b)

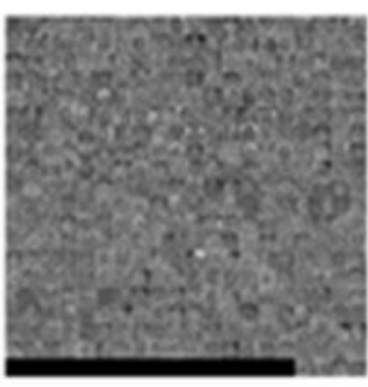

c)

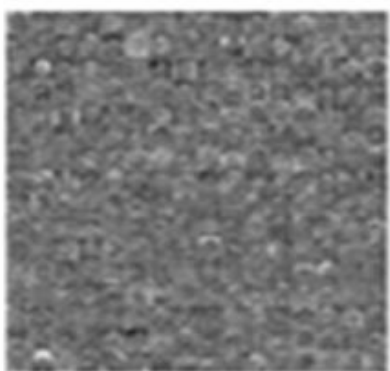

d)

\section{Figure 5}

Features extracted from different convolutional layers of proposed CNN Deep net a) Features extracted from convolutional layer 1 with 64 kernels $b$ ). features extracted from convolutional layer 2 with 128 kernels c)features extracted from convolutional layer 3 with 256 kernels d)features extracted from convolutional layer 4 with 256 kernels.

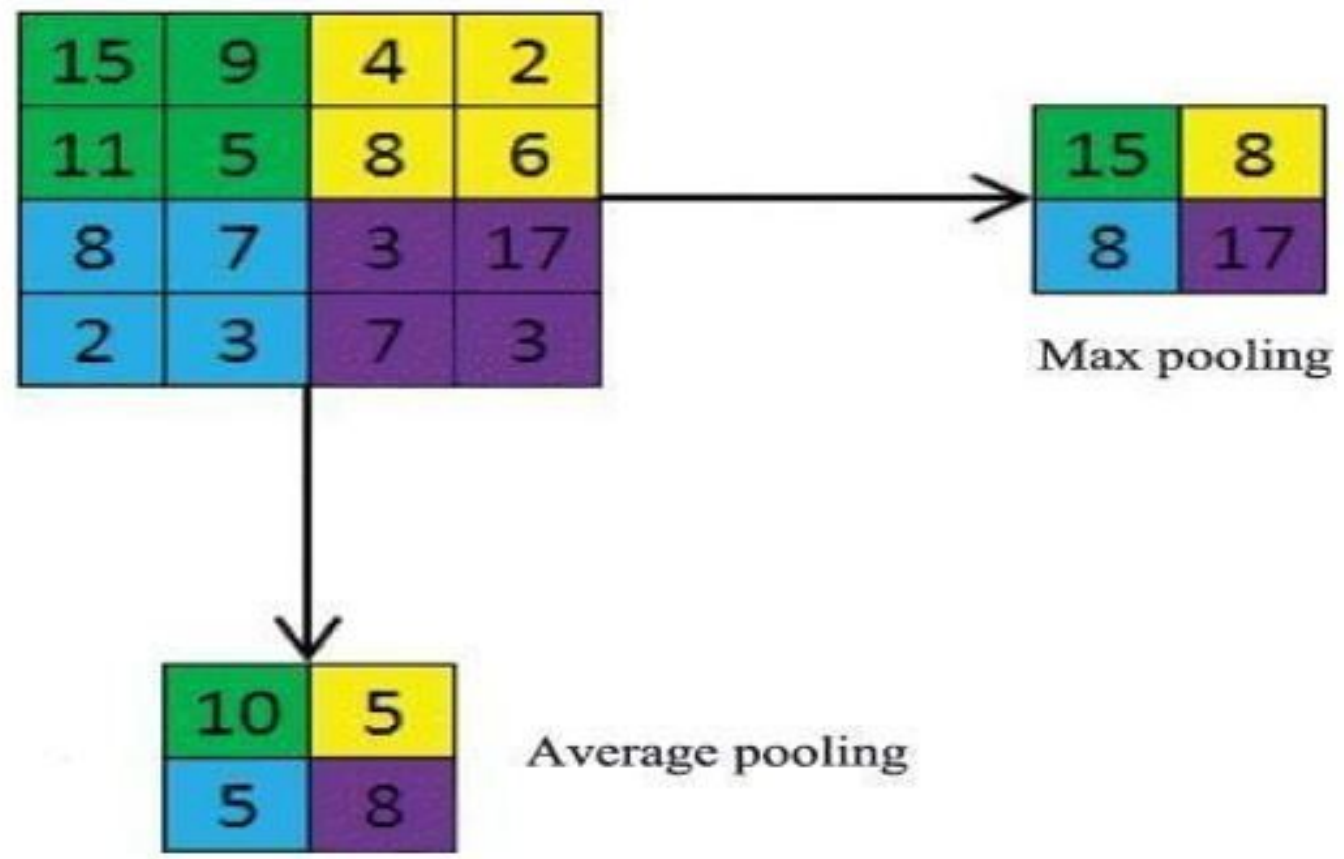

Figure 6

Max and Average pooling 


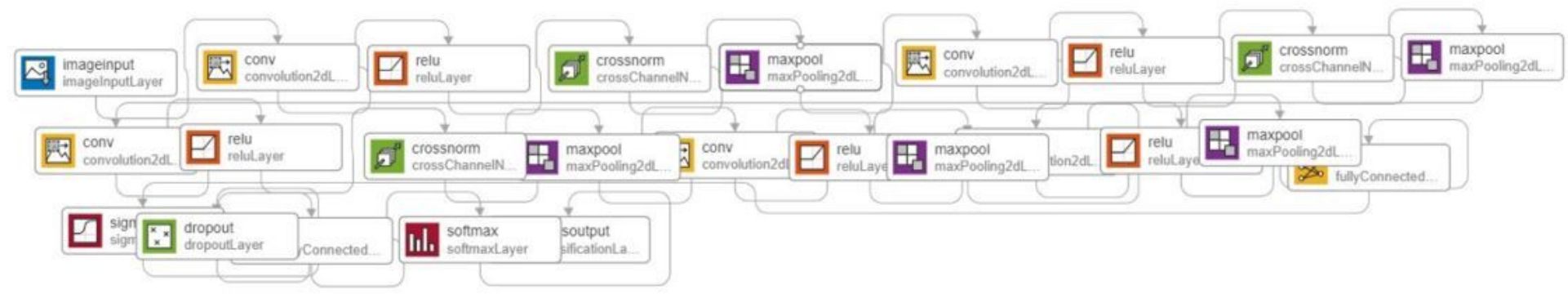

Figure 7

The flow of proposed method of CNN Deep net methodology for classification of tumor and non tumor brain MRI images

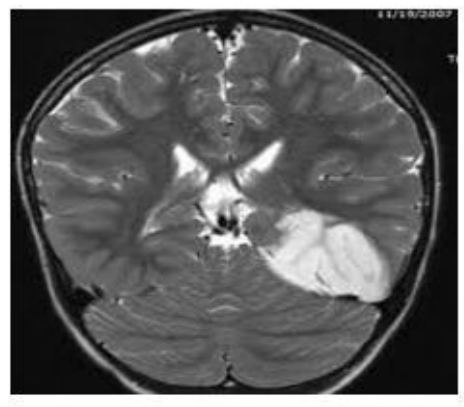

a)

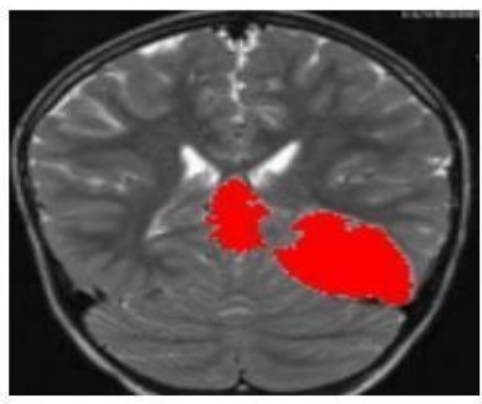

b)

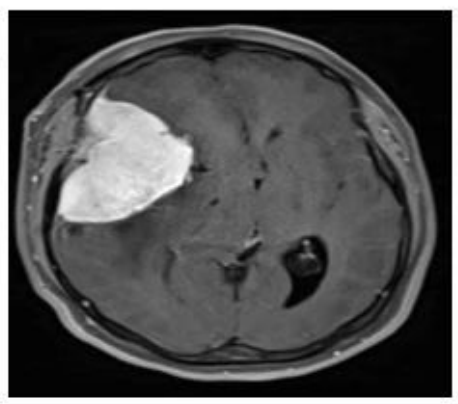

c)

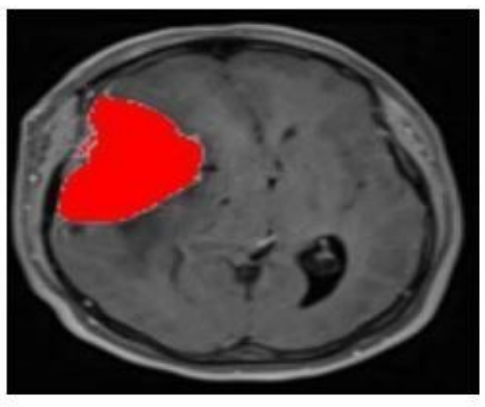

d)

Figure 8

(a) Input MRI (b) Threshold image (c) Morphological area processed image (d) segmented image

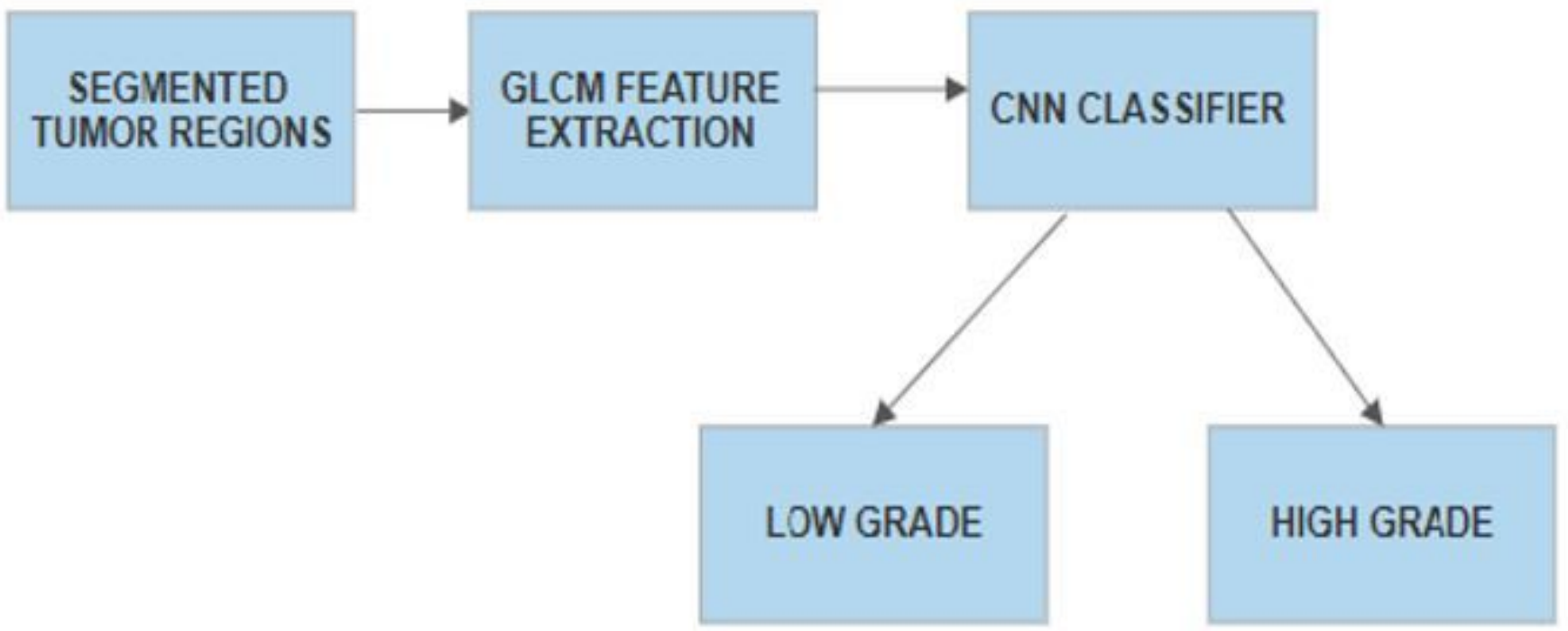


Figure 9

Proposed brain tumor diagnosis system

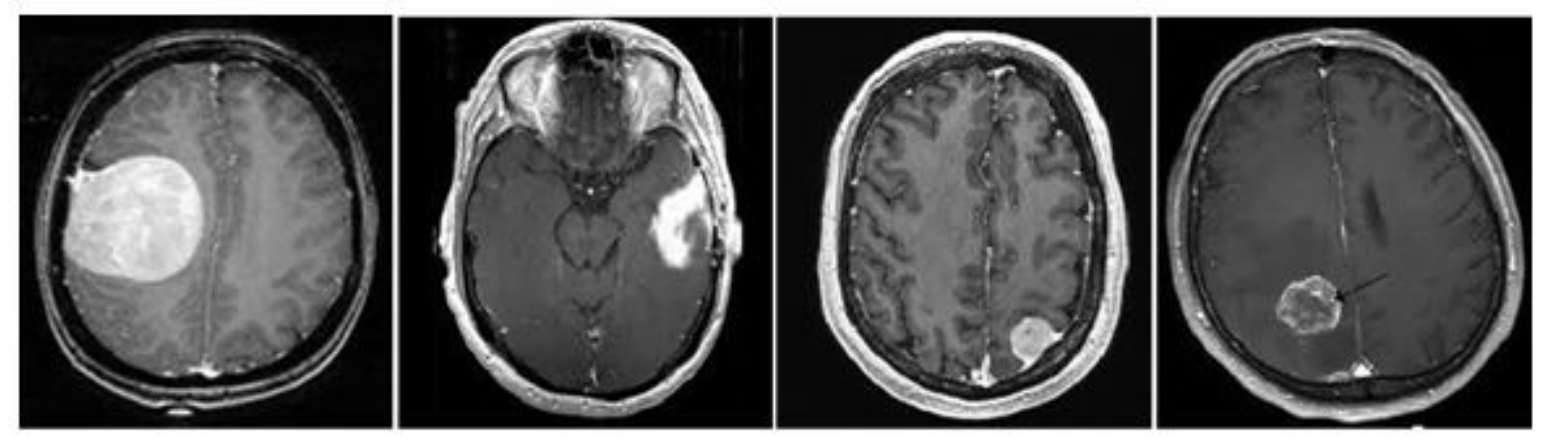

a.low grade meningioma

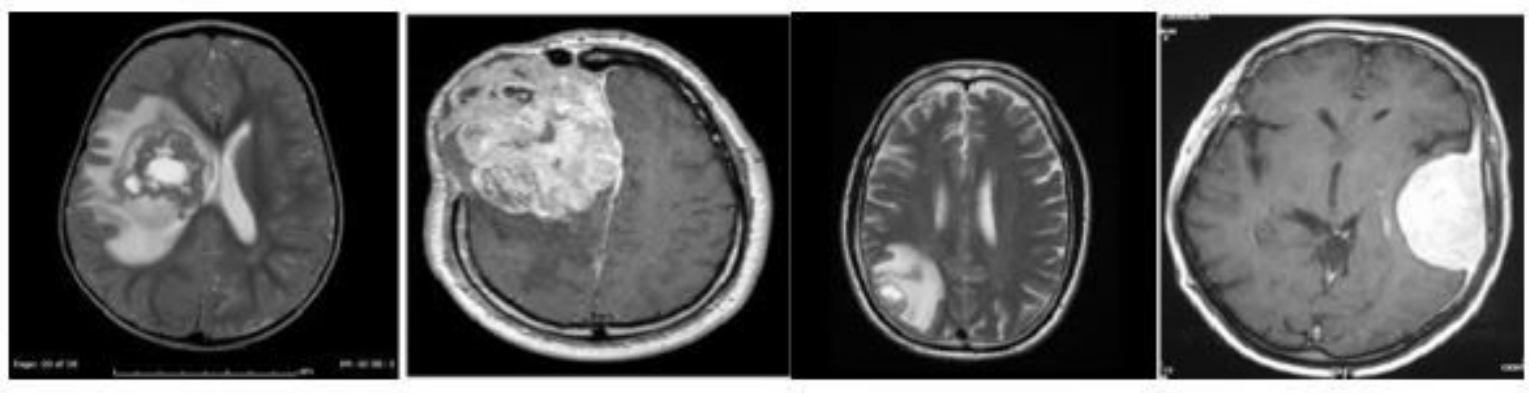

b.high grade meningioma

Figure 10

Classification results of the proposed CNN Deep net a) low grade tumor b) high grade tumor 


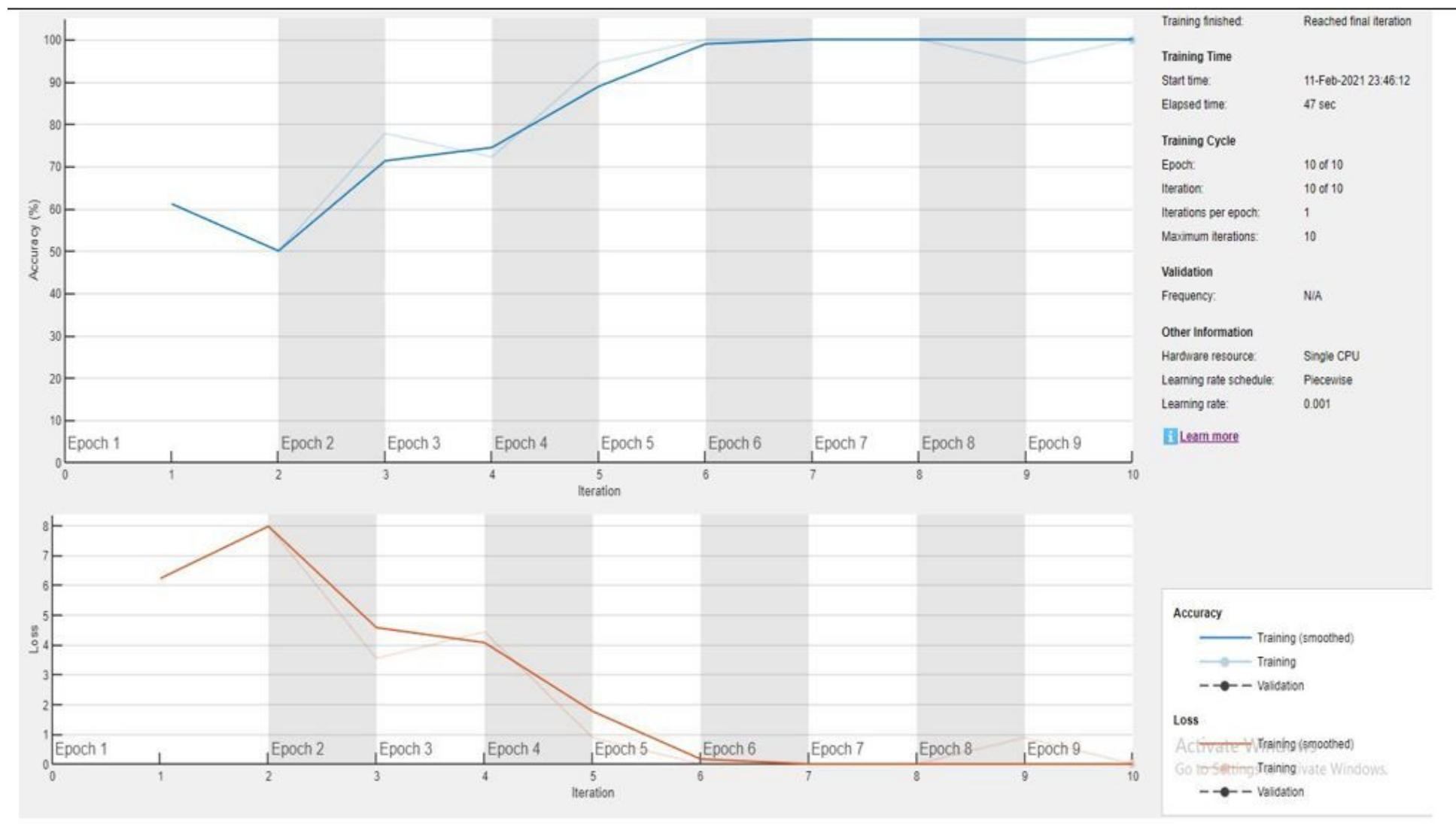

Figure 11

Performance analysis of proposed CNN Deep net for classification of brain tumors images with different epoch

\section{Supplementary Files}

This is a list of supplementary files associated with this preprint. Click to download.

- BIBChecklist1.docx 